\title{
An Aqueous Ammonia Sensor Based on Printed Indium Tin Oxide Layer
}

\author{
Seokhwan Lee, Jung-Yeul Jung, ${ }^{1}$ Moonjin Lee, ${ }^{2}$ and Jiho Chang ${ }^{3 *}$ \\ Department of Convergence Study on the Ocean Science and Technology, \\ Ocean Science and Technology (OST) School, Korea Maritime and Ocean University, \\ Busan 606-791, Republic of Korea \\ ${ }^{1}$ Technology Center for Offshore Plant Industries, Korea Research Institute of Ships and Ocean Engineering, \\ KIOST, Daejeon 305-343, Republic of Korea \\ ${ }^{2}$ Maritime Safety Research Division, Korea Research Institute of Ships and Ocean Engineering, \\ KIOST, Daejeon 305-343, Republic of Korea \\ ${ }^{3}$ Major of Electronic Material Engineering, Korea Maritime and Ocean University, \\ Busan 606-791, Republic of Korea
}

(Received March 29, 2016; accepted July 12, 2016)

Keywords: indium tin oxide (ITO), sensor, hazardous and noxious substance (HNS), ammonia sensor

We proposed an indium tin oxide (ITO) sensor for detecting $\mathrm{NH}_{3}$ in seawater and carried out a series of experiments to investigate the feasibility of using it as a hazardous and noxious substance (HNS) sensor. The ITO layer revealed a distinct resistance change, which was linearly correlated with the $\mathrm{NH}_{3}$ concentration at room temperature. The ITO layer was evaluated using a seawater and ammonium hydroxide $\left(\mathrm{NH}_{4} \mathrm{OH}\right)$ solution. The evaluation revealed an almost linear output signal up to an $\mathrm{NH}_{3}$ concentration of $5 \%$. The sensitivity of the sensor showed a small temperature dependence within a $5-35{ }^{\circ} \mathrm{C}$ range. In addition, no significant degradation was observed during a 7-d-long durability test. The aqueous ammonia sensor based on a printed, porous ITO layer has been explained in terms of reduction reaction at the surface and the formation of an electrical double layer (EDL).

\section{Introduction}

Accidents involving spills of hazardous and noxious substances (HNSs) in the ocean have been recognized as a critical problem for marine life, as a sequence of damage to amenities, and an interference with other legitimate uses of the sea. ${ }^{(1)}$ Unlike oil spills, however, the issue of HNS spills has not received much attention thus far. Two basic means of HNS detection are spectroscopic methods and direct chemical reaction on sensor surfaces. However, neither of these methods provides high spatial resolution and tracking ability, although these issues are essential for minimizing the damage due to HNS spills in the ocean. One possible solution is to establish a floating sensor network around dangerous areas on the ocean surface. Hence, a lightweight and mass-producible sensor is necessary for developing an advanced HNS prevention system.

We focused on ammonia $\left(\mathrm{NH}_{3}\right)$ among HNSs because it is one of the most transported substances in the ocean and commonly used in many commercial products. ${ }^{(2)}$ Concentrated ammonia is caustic, hazardous, colorless, and has pungent odor as well.(3) In addition, ammonia "Corresponding author: e-mail: jiho_chang@kmou.ac.kr http://dx.doi.org/10.18494/SAM.2017.1375 
creates environmental pollution by forming a smog and collapsing the nitrogen cycle. Hence, ammonia detection in the ocean is of great importance. Various technologies have been studied thus far for the detection of ammonia. Various sensors such as electrochemical gas sensors, ${ }^{(4)}$ chemiresistor sensors, ${ }^{(5)}$ chemically modified field-effect transistor (CHEMFET) sensors, ${ }^{(6)}$ optical fiber sensors, ${ }^{(7)}$ and metal oxide semiconductors sensors ${ }^{(8-11)}$ have attracted substantial attention for the monitoring of ammonia in the gaseous phase to provide an easy and fast means of detection. Among these sensors, metal oxide sensors are of particular interest to the authors of this paper because compact, less power consuming, inexpensive, and environmentally friendly sensors may be possible. ${ }^{(12)}$ Metal oxides such as $\mathrm{SnO}_{2}, \mathrm{TiO}_{2}, \mathrm{WO}_{3}, \mathrm{ZnO}$, and $\mathrm{In}_{2} \mathrm{O}_{3}$ have been investigated for various sensor applications. ${ }^{(8-12)}$

In this study, we have investigated the feasibility of using a printed indium tin oxide (ITO) layer as a new HNS detector. The printing procedure has been investigated and the basic sensor performance was evaluated. The robustness of the ITO layer has also been tested.

\section{Experiments}

We fabricated a paste for printing that contains ITO powder $\left(90 \% \mathrm{In}_{2} \mathrm{O}_{3}, 10 \% \mathrm{SnO}_{2}\right.$, the mean diameter was $38.5 \mathrm{~nm}$ with the standard deviation of $5.8 \mathrm{~nm}$ ) and an organic binder (ethyl cellulose and $\alpha$-terpineol) at a $1: 1$ ratio by weight. We used a quartz $\left(\mathrm{SiO}_{2}\right)$ substrate, which has a dimension of $35 \times 35 \times 1 \mathrm{~mm}^{3}$. The substrate was cleaned sequentially with methanol, acetone, and DI water in an ultrasonic bath (each step took $15 \mathrm{~min}$ ) to remove organics and impurities on the surface. The ITO powder was printed using a zigzag-pattern mask [thickness: $30 \mu \mathrm{m}$, mesh size: 325 mesh $(45 \mu \mathrm{m})]$ on a quartz substrate. After printing, the ITO layer was heated at $200{ }^{\circ} \mathrm{C}$ for $1.5 \mathrm{~h}$ to remove the volatile organic solvent in the printed layer (debinding process). We have optimized the viscosity of the paste in terms of the transfer ratio, which means the ratio between the linewidth of the original pattern and the printed result. To monitor the electric signal from the ITO layer, we made contacts at both ends of the ITO layer using a silver paste. The details of the optimization of the printing process were reported previously. ${ }^{(13,14)}$

Field emission scanning electron microscopy (FE-SEM) was used to investigate the surfaces of the as-printed/tested ITO layers. The structural properties of the ITO layer were characterized by $\mathrm{X}$-ray diffraction (XRD). The sensor operation was monitored using a current-voltage source. We recorded the resistance of the sensor in air as a reference signal and monitored variations caused by soaking in seawater and ammonium hydroxide solutions. We monitored the temporal variations of resistance in the air and soaked in the solutions. We used four different solutions: seawater, $5 \% \mathrm{NH}_{3}$ in seawater, $10 \% \mathrm{NH}_{3}$ in seawater, and $20 \% \mathrm{NH}_{3}$ in seawater. A floating platform was necessary to position the sensor on the liquid surface. The ITO layer was placed just beneath the platform. $I-V$ characteristics were measured under a DC bias of 5V using a Keithley 2400V instrument.

\section{Results and Discussion}

To investigate the feasibility of the ITO layer as HNS sensor, we monitored the resistance change of the ITO layer in accordance with the various liquids as shown in Fig. 1. First, to estimate the reference signal level $\left(R_{\text {air }}\right)$, the resistance of the ITO layer was monitored in air for $100 \mathrm{~s}$, after which the ITO layer was soaked in the solutions. When it was soaked, the resistance of the ITO layer $\left(R_{\text {liq }}\right)$ dropped abruptly and then slowly increased to a specific value for all the samples. 


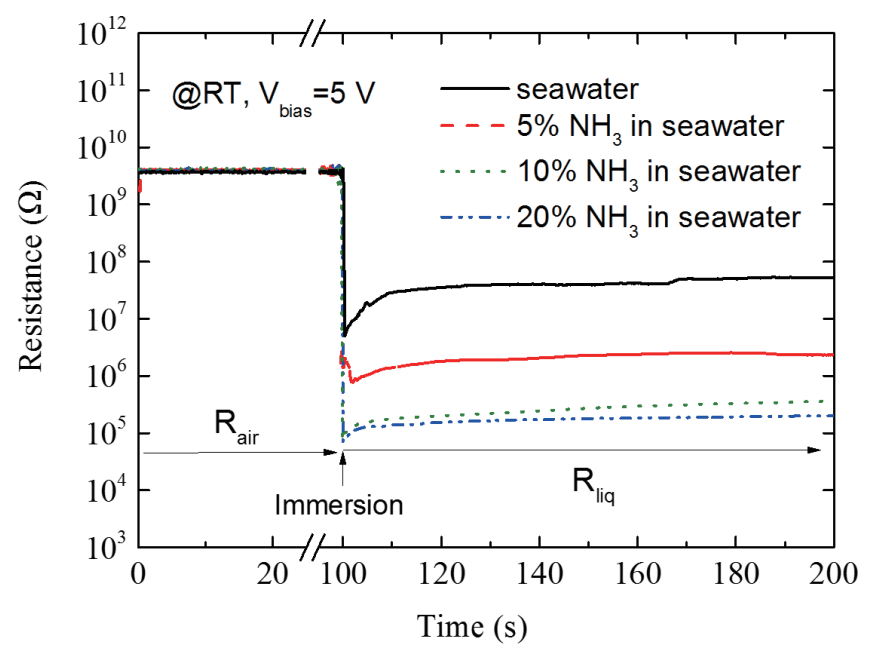

Fig. 1. (Color online) Time-resolved resistance change of the ITO layers soaked in various solutions.

The measured resistance change ranges over $2-4$ orders of magnitude as a function of concentration. The reference signal $\left(R_{\text {air }}\right)$ of the ITO layer was about $4.0 \pm 0.1 \times 10^{9} \Omega$. The minimum resistances $\left(R_{\text {liq }}\right)$ for each solution were dropped to $4.9 \times 10^{6} \Omega\left(0 \% \mathrm{NH}_{3}\right.$ in seawater $)$, $8.4 \times 10^{5} \Omega\left(5 \% \mathrm{NH}_{3}\right.$ in seawater $), 7.7 \times 10^{4} \Omega\left(10 \% \mathrm{NH}_{3}\right.$ in seawater $)$, and $7.1 \times 10^{4} \Omega(20 \%$ $\mathrm{NH}_{3}$ in seawater). The sensors were maintained in the solutions without any agitation; however, the resistance increased to certain values, which was also related to the $\mathrm{NH}_{3}$ concentration of the solution: $4.8 \times 10^{7} \Omega\left(0 \% \mathrm{NH}_{3}\right.$ in seawater $), 1.6 \times 10^{6} \Omega\left(5 \% \mathrm{NH}_{3}\right.$ in seawater $), 2.0 \times 10^{5} \Omega(10 \%$ $\mathrm{NH}_{3}$ in seawater), and $1.5 \times 10^{5} \Omega\left(20 \% \mathrm{NH}_{3}\right.$ in seawater). Figure 1 clearly implies that the printed ITO layer can be used for ammonium hydroxide detection in seawater, since both initial and saturated resistance values are closely related to the concentration of the solution.

Here, it should be mentioned that when large amounts of HNSs spill into the ocean, the high concentrations of HNSs might not be maintained over a long time, because the physical and chemical reactions of the ocean will dilute the substances. ${ }^{(15)}$ Consequently, the highest residual HNS concentration could be estimated to be no more than $\sim 10 \%$. Therefore, we have evaluated the sensor performance within the estimated range of likely concentration.

The resistance change of the ITO layer (shown in Fig. 1) can be simply explained by a redox reaction and the electrical double layer (EDL) formation. Generally, redox reactions (oxidationreduction reactions) are simultaneous reactions, meaning that they cannot occur independently. Oxidation is the loss of electrons during a reaction and reduction is the gain of electrons during a reaction. Owing to the change in free electron density, the conductivity of a material is changed as a result of redox. In our experiment, when an ITO layer was soaked in a solution, the resistance dropped rapidly. This means that the free electron density at the surface increased as a result of the reduction. When the ITO layer is soaked in an aqueous solution, the desorption of oxygen from the surface takes place as described in the following reduction reaction: ${ }^{(16,17)}$

$$
\mathrm{R}+\mathrm{O}^{-}(\mathrm{ads})=\mathrm{RO}+\mathrm{e}^{-}
$$


where " $\mathrm{R}$ " indicates a reducing agent. $\mathrm{O}^{-}$(ads) means adsorbed oxygen atom on the surface. RO and $\mathrm{e}^{-}$indicate a product of reduction and free electron, respectively. The reducing agent could be ammonia or electrolytes in seawater. As one can see, however, the resistance change is closely related to the concentration of ammonia. Hence, we considered that the dominant reducing agent might be the ammonia in this experiment. As a consequence of this reaction, electrons are liberated, and the carrier concentration of the ITO layer increases. Therefore, the resistance of the ITO layer decreases abruptly, as shown in Fig. 1.

Note that one should also consider the effect of electrolytes in the seawater. Thus, the net effect of ammonia itself should be expressed as the difference between the resistance of an $\mathrm{NH}_{3}$ solution and that of the seawater.

Since the reaction happens in an electrolyte, an electrical double layer (EDL) is also formed near the sensor surface. ${ }^{(18,19)}$ It is well known that the EDL consists of two layers; the first layer (the stern layer) comprises counter ions adsorbed onto the surface due to chemical interactions, and the second layer (the diffuse layer) is composed of ions attracted to the surface charge via coulombic force, which electrically screens the first layer. This second layer is loosely associated with the surface. The formation of an EDL produces an equilibrium state near the surface, and further resistance changes will be hampered as shown in Fig. 1.

Figures 2(a) and 2(b) reveal the responses of the ITO layer. The sensitivity $S$ is defined as $S=\left(R_{\text {air }}\right.$ $\left.-R_{\text {liq }}\right) / R_{\text {air }}$, where $R_{\text {air }}$ and $R_{\text {liq }}$ are the resistances of the ITO layer in air and liquid, respectively. $R_{\text {liq }}$ is defined as the resistance measured at $200 \mathrm{~s}$ after immersion.

As the $\mathrm{NH}_{3}$ concentration increased, the sensitivity $\mathrm{S}$ also increases [Fig. 2(a)]. A linear response was obtained up to an $\mathrm{NH}_{3}$ concentration of $5 \%$; however, the saturation of the sensitivity was observed in the high-concentration region. Rout et al. have observed a similar phenomenon from $\mathrm{SnO}_{2}$ - and $\mathrm{In}_{2} \mathrm{O}_{3}$-based $\mathrm{NH}_{3}$ gas sensors. ${ }^{(16)}$ They observed that the $\mathrm{SnO}_{2}$-based sensors revealed comparably greater nonlinearity and higher sensitivity as well. They mentioned that the nonlinear response was caused by the higher carrier concentration in the $\mathrm{SnO}_{2}$ layer, and it is related

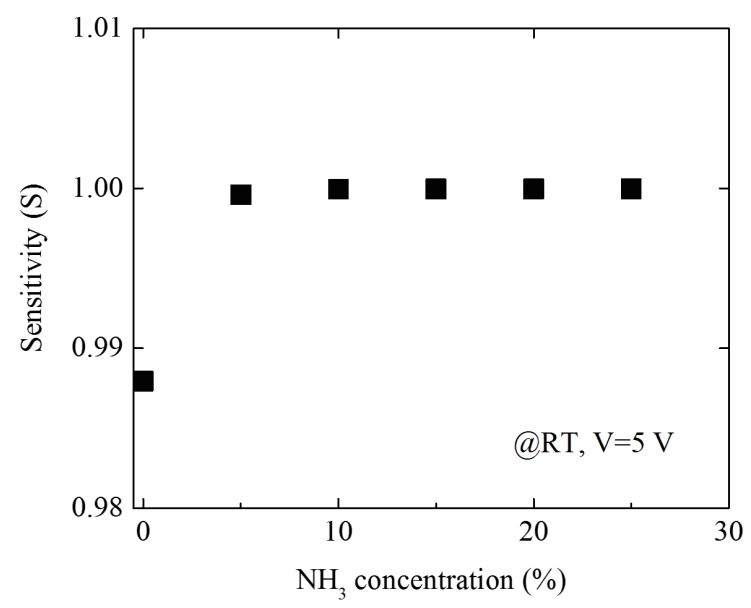

(a)

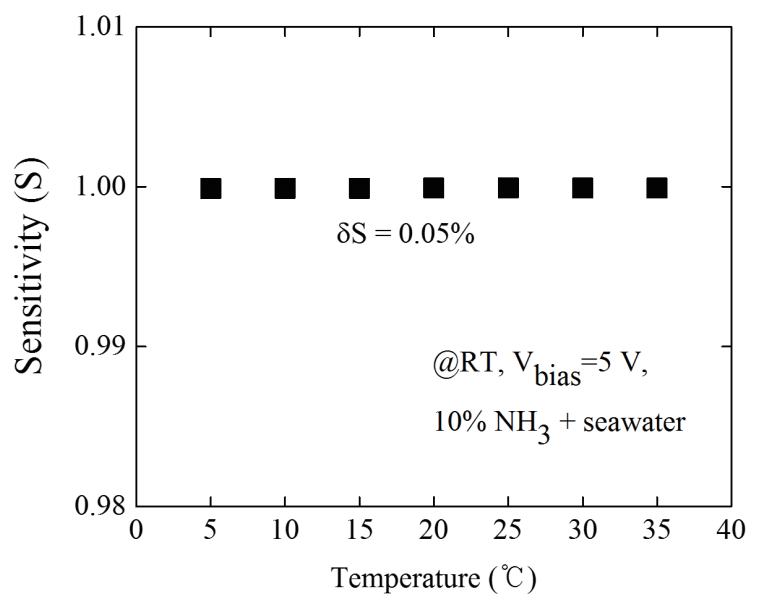

(b)

Fig. 2. Sensitivity change of the ITO sensors along with (a) different $\mathrm{NH}_{3}$ concentrations and (b) different seawater temperatures. 
to the grain size of it. In our experiment, since we just used one kind of ITO powder, the grain size effect might be negligible. Therefore, we temporally attributed the sensitivity saturation to the high intrinsic carrier concentration of ITO.

Figure 2(b) shows the sensitivity variation along with an ambient temperature change. Note that it is an important factor for HNS sensor applications, because the surface temperature of the ocean varies from 0 (zero) to $30^{\circ} \mathrm{C}$ according to the position on Earth and the season as well. Hence, the sensitivity of a sensor is also strongly correlated to ambient temperature. When the ambient temperature varied from 5 to $35{ }^{\circ} \mathrm{C}$, the sensitivity varied less than $0.05 \%$, which is sufficiently small to ignore the temperature compensation for the practical use of an ITO sensor.

We have examined the robustness and stability of the ITO layer, because seawater is a harsh environment for any device. We have performed a 7-days-long continuous operation test (under DC $5 \mathrm{~V}$ bias) with the ITO sensor. Figures 3(a) and 3(b) show the XRD and FE-SEM images (insets) of the as-printed and tested ITO sensors. Figure 3(a) shows the cubic bixbyite phase of an as-printed ITO layer with an intense (222) peak at 30.6 degree. The strong diffraction peaks were assigned to (222), (400), (440), and (622) planes of the cubic bixbyite ITO (JCPDS 01-089-4198). No significant change was observed from both XRD and FE-SEM results as shown in Fig. 3(b).

Further investigation has performed to confirm the stability of the ITO sensor. We have compared both sensitivity and related surface morphology change of ITO- and ZnO-based sensors

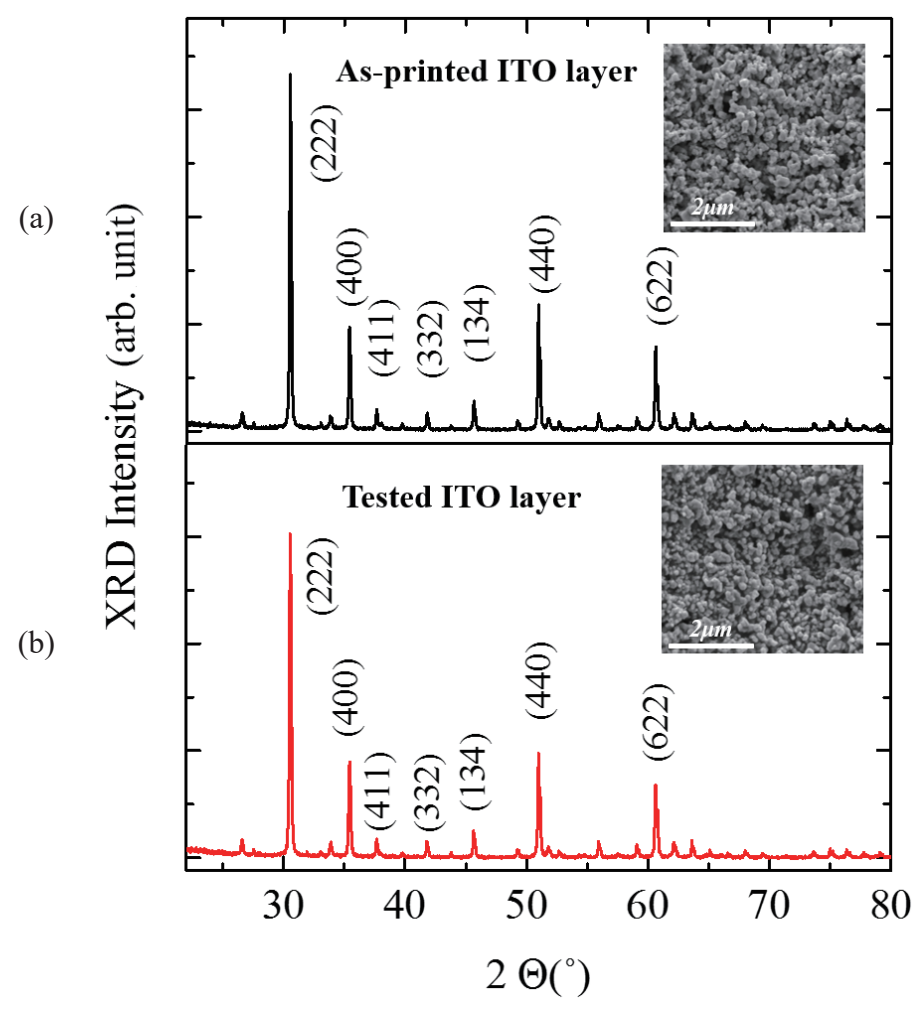

Fig. 3. (Color online) XRD pattern and FE-SEM images (insets) of (a) as-printed ITO layer and (b) tested ITO layer. 


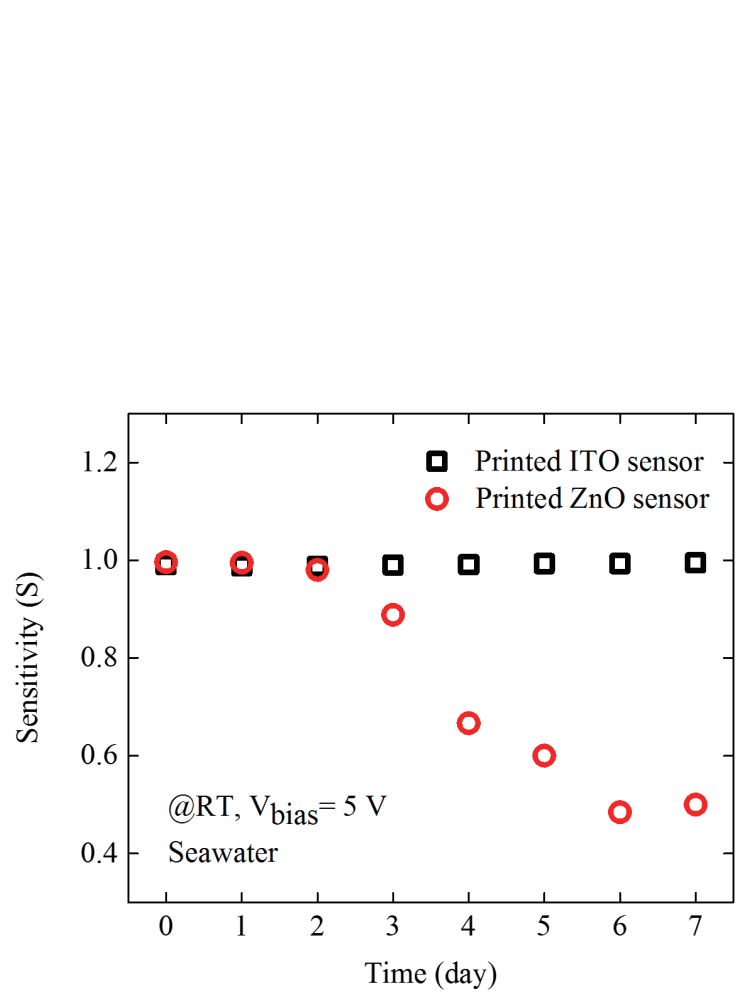

(a)

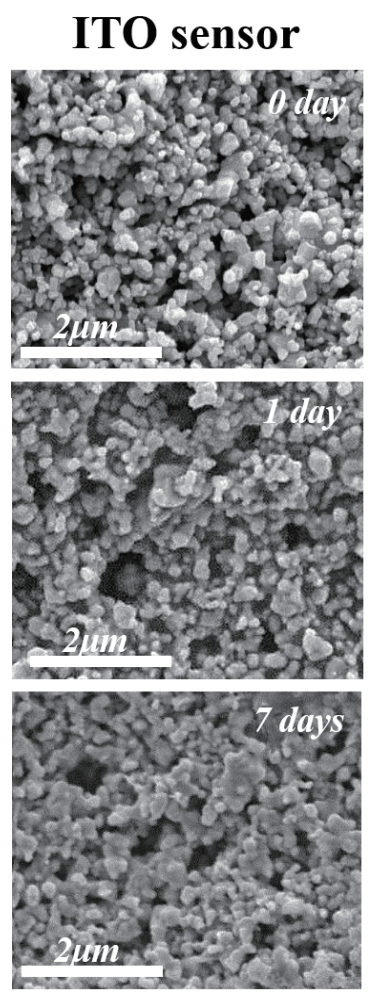

(b)
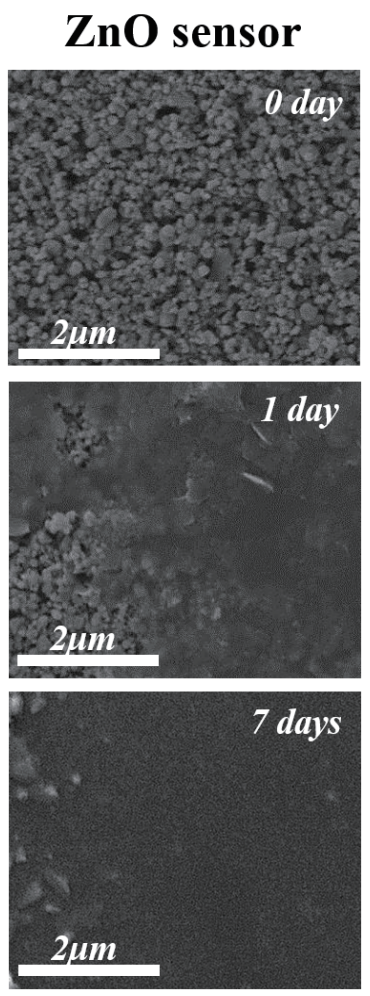

(c)

Fig. 4. (Color online) (a) Continuous operating test results for the ITO and $\mathrm{ZnO}$ sensors during 7 days at room temperature. SEM images of the (b) ITO sensor surface and (c) ZnO sensor surface during the test.

for 7 days under DC $5 \mathrm{~V}$ bias. Note that the $\mathrm{ZnO}$ sensor was fabricated by the same method, and had the same dimensions with the ITO sensors. We found that the $\mathrm{ZnO}$ sensor could not endure long-term operation in seawater. Figure 4(a) shows the sensitivity variation of both sensors. The sensitivity of the $\mathrm{ZnO}$ sensor was decreased abruptly after just 2 days of operation. However, no considerable degradation was observed from the ITO sensor. Figures 4(b) and 4(c) show the morphological evolution of both sensors. A dramatic change in surface morphology was observed from the $\mathrm{ZnO}$ sensor [Fig. 4(c)]. Those results indicate that the ITO sensor is more suitable for a HNS application in the ocean in terms of chemical stability.

\section{Conclusions}

The feasibility of using a printed ITO layer for HNS detection has been investigated. The screen-printed ITO layer has a porous structure, which is preferred for developing a highly sensitive sensor. Sensor operation was demonstrated by monitoring the time-resolved resistance change of the ITO layer. When the ITO sensor is soaked in the solution, resistance varied linearly according to the ammonium hydroxide concentration $(<5 \%)$. The temperature dependence of the sensitivity has been evaluated within the range of sea surface temperature $\left(5-30^{\circ} \mathrm{C}\right)$. A negligible change was 
observed, which means that sensitivity compensation by an external driving circuit is unnecessary. The robustness of the ITO layer has been confirmed by the continuous operation test ( 7 days) in seawater. Both the sensitivity and surface morphology of the ITO sensor revealed negligible degradation within our experimental range.

\section{Acknowledgements}

This research was part of the project entitled 'Development of Management Technology for HNS Accident', funded by the Ministry of Oceans and Fisheries, Korea.

\section{References}

1 I. Cunha, S. Moreira, and M. M. Santos: J. Hazard. Mater. 285 (2015) 509.

2 Are HNS Spills More Dangerous than Oil Spills?: ITOPF, http://www.itopf.com/fileadmin/data/Documents/ Papers/interspill09_hns.pdf (accessed May 2014).

3 Toxic FAQ Sheet for Ammonia: Agency for Toxic Substances and Disease Registry, http://www.atsdr.cdc. gov/tfacts 126.pdf (accessed September 2004)

4 Y. Chao, W. J. Buttner, K. Gupta, W. R. Renrose, and J. R. Stetter: Chemical Sensors VI: Chemical and Biological Sensors and Analytical Methods (The Electrochemical Society proceeding, 2004-8) p. 109.

5 C. K. Ho and C. F. Lohrstorfer: Groundwater Monit. Rem. 23 (2003) 85.

6 T. V. Krishna, J. R. Jessing, D. D. Russell, J. Scaggs, L. R. Warner, and J. A. Hartman: Proc. 15th Biennial University/Government/Industry Microelectronics Symposium (IEEE, 2003) p. 271.

7 W. Cao and Y. Duan: Sens. Actuators, B 110 (2005) 252.

8 W.-C. Tian, Y.-H. Ho, C.-H. Chen, and C.-Y. Kuo: Sensors 13 (2013) 865.

9 Y.-D. Wang, Z.-X. Chen, Y.-F. Li, Z.-L. Zhou, and X.-H. Wu: Solid-State Electron. 45 (2001) 639.

10 J. Y. Park, D. E. Song, and S. S. Kim: Nanotechnology 19 (2008) 105503.

11 C. Li, D. Zhang, X. Liu, S. Han, T. Tang, J. Han, and C. Zhou: Appl. Phys. Lett. 82 (2003) 1613.

12 A. Vaseashta, M. Vaclavikova, S. Vaseashta, G. Gallios, and P. Roy: Sci. Technol. Adv. Mater. 8 (2007) 47.

13 S. H. Lee, J. Koo, S. Jung, M. Lee, J. Y. Jung, and J. Chang: Proc. 5th Asia Pacific Optical Sensors Conference (International Society for Optics and Photonics, 2015) p. 96553E.

14 J. Koo, J. Y. Jung, M. Lee, and J. Chang: Mar. Pollut. Bull. 98 (2015) 130.

15 Hazardous and Noxious Substances (HNS) Chemical Fate and Effects: ITOPF, http://www.itopf.com/ knowledge-resources/documents-guides/hazardous-and-noxious-substances-hns/chemical-fate-and-effects/ (accessed May 2014).

16 C. S. Rout, M. Hegde, A. Govindaraj, and C. N. R. Rao: Nanotechnology 18 (2007) 205504.

17 K. K. Makhija, A. Ray, R. M. Patel, and U. B. Trivedi: Bull. Mater. Sci. 28 (2005) 9.

18 L. L. Zhang and X. S. Zhao: Chem. Soc. Rev. 38 (2009) 2520.

19 K. Bourikas, C. Kordulis, J. Vakros, and A. Lycourghiotis: Adv. Colloid Interface Sci. 110 (2004) 97. 